SSC19-WKVI-04

\title{
Modeling and Simulation of a Long-Wave Infrared Polarimetric Sensor for Space Object Detection and Characterization
}

\author{
Kevin Pohl, Jonathan Black, Jonathan Pitt, Edward Colbert \\ Virginia Polytechnic Institute and State University \\ 900 N Glebe Rd, Arlington, VA, 22203; 443-540-0288 \\ kpohl@vt.edu
}

\begin{abstract}
Long-Wave Infrared (LWIR, wavelength $>8$ um) polarimetric measurements can be used to characterize space objects under certain conditions. Both visible and LWIR polarimetry have been demonstrated extensively in terrestrial applications for characterization and detection of objects of interest. Visible polarimetry has also been demonstrated for space object detection. A simulation of a camera and telescope for collection of LWIR polarimetric signatures of space objects has been assembled using three software packages: Systems Tool Kit (STK), MATLAB, and FRED. Characterization of space objects is generally possible across a wide range of target surface temperatures and emissivities, and at a sub-pixel level; characterization is reliable in a narrower range. This approach represents an initial step forward in optical systems for space situational awareness in that it offers a wider field of view than equivalently sized visible light collectors, and it can be used both day and night, regardless of target illumination.
\end{abstract}

\section{INTRODUCTION}

The objective of the project, the opening stage of which is documented here, is to explore a new avenue for space situational awareness (SSA). Today, SSA is maintained through a combination of ground sensors (radars, telescopes) and voluntary sharing of telemetry and other information with various organizations that maintain active catalogs of space objects. The present work seeks to demonstrate, initially through modeling and simulation, the characterization of low Earth orbit (LEO) satellites by a long-wave infrared polarimetric imaging system.

Long-wave infrared polarimetry has been demonstrated for man-made object detection in a variety of settings. ${ }^{1,2}$ In addition, visible spectrum polarimetry has been demonstrated for detection and identification of satellites in geosynchronous orbit GEO. ${ }^{3,4}$ Speicher used visible light polarimetry to detect and identify GEO satellites. The experimental setup only measured S0 and S1, and due to the dimness of the targets required an integration time of $\sim 20$ seconds. Repeated observations over time revealed differences in signature between individual satellites, both between different types of vehicles, and between vehicles of the same design, but of different age. The latter effect is of particular interest, as it is the material properties of the surface layer (e.g. paint) that drive the complex index of refraction and thus the polarization signature. Those material properties change over time due to exposure to the space environment. ${ }^{4}$ Further work has shown that the polarization signatures of individual components (e.g. dish antenna, bus, solar panels) sum together to create a composite signature. ${ }^{5,6}$
Passive long-wave infrared polarimetry for man-made object detection has been the subject of numerous studies since at least the 1990s. ${ }^{1,2,7}$ The advantage of long-wave infrared for these purposes is that it measures primarily the target's self-emission, though emission by nearby sources (e.g. low clouds), and thus reflection off the target, can interfere. This occurs because the reflected light is polarized perpendicularly to the emitted light, resulting in reduced values for $\mathrm{S} 1$ and $\mathrm{S} 2,7,8$ and thus a reduced signal-to-noise ratio. For space object detection this is not a concern, as space objects are generally not close to each other, and solar radiation incident on the target (and resulting reflected radiation) in the 8-9 micron band is an order of magnitude or more less than the self-emission of the target.

Ground target detection using long-wave infrared polarimetry is effective both day and night. An object viewed from a specific angle will remain virtually unchanged in terms of degree of linear polarization (DOLP) regardless of changes in thermal contrast with its surroundings. ${ }^{1}$

There has also been some work done concerning longwave infrared (without polarization) for detection of space objects, beginning in the late 1980s. Targets included geosynchronous satellites, which could not be resolved, but could be detected..$^{9,10,11}$ Studies have also been conducted using space based LWIR sensors to detect and characterize space objects. ${ }^{12}$ In both cases detection was feasible during both daytime and nighttime. The tradeoff between visible light collection and LWIR is one of resolution against collection 
opportunities. When using a long-wave IR sensor, the target is its own source of illumination, where a visible light sensor requires an external source of illumination (e.g. the Sun).

\section{Polarization measurement}

Several methods exist to measure the polarization of light, Jones matrices, ${ }^{13}$ coherency matrices, ${ }^{14}$ Mueller matrices, and others. ${ }^{15}$ Of these methods, Mueller calculus is most appropriate for use with polarimeters, where the polarization state of a light beam is described by the Stokes Vector $\mathbf{S}$ and the polarization-altering characteristics of a target are described by the Mueller matrix M. The Stokes vector is based on six flux measurements using ideal polarizers in front of a radiometer: horizontal $\left(\mathrm{P}_{\mathrm{H}}\right)$, vertical $\left(\mathrm{P}_{\mathrm{V}}\right)$, diagonal (45 and 135 degrees; $\mathrm{P}_{45}$ and $\mathrm{P}_{135}$, respectively), and left $\left(\mathrm{P}_{\mathrm{L}}\right)$ and right circular $\left(\mathrm{P}_{\mathrm{R}}\right){ }^{15}$

The Stokes vector is then defined as

$$
\boldsymbol{S}=\left[\begin{array}{c}
S_{0} \\
S_{1} \\
S_{2} \\
S_{3}
\end{array}\right]=\left[\begin{array}{c}
P_{H}+P_{V} \\
P_{H}-P_{V} \\
P_{45}-P_{135} \\
P_{R}-P_{L}
\end{array}\right]
$$

where $s_{0}, s_{1}, s_{2}$, and $s_{3}$ are the Stokes vector components in units of watts per meter squared. The Stokes vector represents an average over area, solid angle, and wavelength. ${ }^{15}$ From the Stokes vector four polarization parameters can be determined: ${ }^{14}$

$$
\text { Flux } \quad P=s_{0}
$$

$$
\begin{array}{ll}
\begin{array}{l}
\text { Degree of } \\
\text { polarization }
\end{array} & D O P=\frac{\sqrt{s_{1}^{2}+s_{2}^{2}+s_{3}^{2}}}{s_{0}} \\
\begin{array}{l}
\text { Degree of linear } \\
\text { polarization }
\end{array} & D O L P=\frac{\sqrt{s_{1}^{2}+s_{2}^{2}}}{s_{0}} \\
\begin{array}{l}
\text { Degree of circular } \\
\text { polarization }
\end{array} & D O C P=\frac{s_{3}}{s_{0}}
\end{array}
$$

Of these flux and degree of linear polarization are most relevant for the present study. The bulk of the materials encountered-dielectrics, metals, and thin films (coatings, paints)-have negligible rates of circular polarization, ${ }^{15}$ reducing the value of DOP and DOCP measurements.

\section{TOOLS}

\section{Systems Tool Kit (STK)}

STK provides a sophisticated modeling environment to model space systems and evaluate their performance. For this project STK provides the ability to model the positions and attitudes of a satellite with respect to a notional ground-based telescope over time: range to target and angle from which the satellite is seen by the telescope. In addition, the EOIR (electrooptical/infrared) toolkit can provide simulated imagery based on the selected parameters for sensor and target, though it is not capable of simulating polarimetric measurements.

\section{Optical Photonics: FRED}

FRED is a software suite which simulates the propagation of light through any optomechanical system by raytracing, including polarimetric measurements. FRED keeps track of the polarization of the light as it makes its way from the target to the sensor.

\section{MODELING AND SIMULATION}

The range of temperatures and material emissivities for which an object in space could be expected to self-emit substantially more LWIR radiation than it reflects from incoming solar radiation were determined. This is important, because reflected polarization competes with emitted polarization when calculating the Stokes vector components. $^{7}$

To calculate the solar flux on an object at a given wavelength, one must first determine the total spectral radiance of the Sun $\left(b_{T, S u n}\right)$ at a given wavelength:

$$
\begin{aligned}
& p=2 \cdot h \cdot \frac{c^{2}}{\lambda^{5}} \\
& b_{T, \text { Sun }}=p /\left(\exp \left(\frac{h \cdot c}{\lambda \cdot k \cdot T}\right)-1\right)
\end{aligned}
$$

where $h$ is Planck's constant, $c$ is the speed of light, $k$ is Boltzmann's constant, $T$ is the temperature of the Sun, and $\lambda$ is the wavelength. Next, the radiance is multiplied by the square of the ratio of the solar radius to the radius of Earth's orbit and Lambert's cosine Law is applied:

$$
b_{T, \text { Sun@Earthorbit }}=b_{T, \text { Sun }} \cdot\left(2.177 \cdot 10^{-5}\right) \cdot \pi
$$

The calculation for a notional object in low Earth orbit is similar; however, the emissivity of the object in the waveband of interest needs to be factored into the calculation. 


$$
b_{T, O b j}=p /\left(\exp \left(\frac{h \cdot c}{\lambda \cdot k \cdot T}\right)-1\right) \cdot \pi \cdot \varepsilon
$$

where $\varepsilon$ is the emissivity of the object at a given wavelength $\lambda$.

This process is repeated for 200 discrete wavelengths between 8 and 9 microns and the resulting blackbody radiation values are summed across the waveband of interest. A MATLAB script was written to compare selfemission with incoming solar radiation in the 8 to $9 \mu \mathrm{m}$ band. The resulting graph (Figure 1) shows that for all but the most reflective surface materials (e.g. bare, polished metal) even relatively cold objects (below $270 \mathrm{~K}$ ) emit more an order of magnitude more thermal radiation than they receive from the Sun.

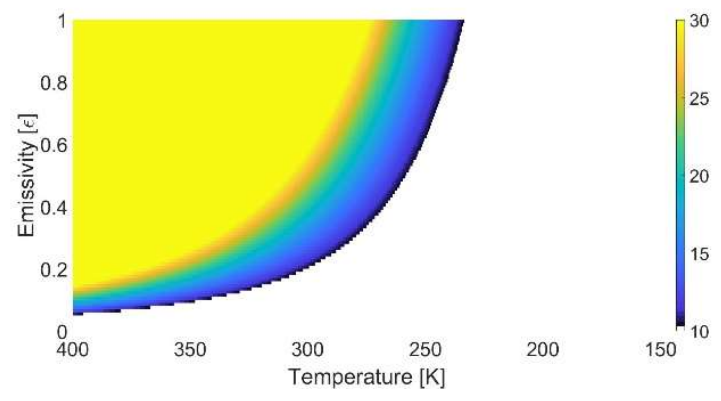

Figure 1: Comparison of self-emission to reflected solar radiation as a function of object emissivity and temperature (the color scale maximum is 30 , but the maximum calculated factor was $>400$ )

Since rejecting waste heat is an important function of a satellite's outer structure, emissivities of 0.8 or more in the LWIR can be expected. In addition, active LEO space objects will spend most of their lifetime on the warm side of $270 \mathrm{~K}$-active satellites in general can be expected to have surface temperatures between $270 \mathrm{~K}$ and $380 \mathrm{~K} .{ }^{10}$ Because the target's self-emission is the dominant contribution in this temperature range, this method allows detection whenever the target is overhead including when the target is in Earth's shadow.

The overall algorithm is a manual workflow using STK to generate range and rotation angle parameters to subsequently use as input in computing polarimetric measurements in FRED. A simplified model the size of the International Space Station (ISS) was placed in an appropriate orbit in STK using publicly available twoline elements, and the orbit was propagated for one month to ensure a wide variety of viewing angles and target distances. Figure 2 shows a view of the orbit track, the target body coordinate system (blue arrows), the vector from the telescope to the target (pink arrow), and the two rotation angles that describe how the target will need to be rotated in FRED to simulate the polarimetric measurement.

STK generates a comma-separated-values file listing all the contacts over the course of a month, along with the magnitude of the distance from the telescope to the target and the rotation angles in 60 second increments. A close overhead pass was selected from this dataset to perform an initial set of simulations in FRED.

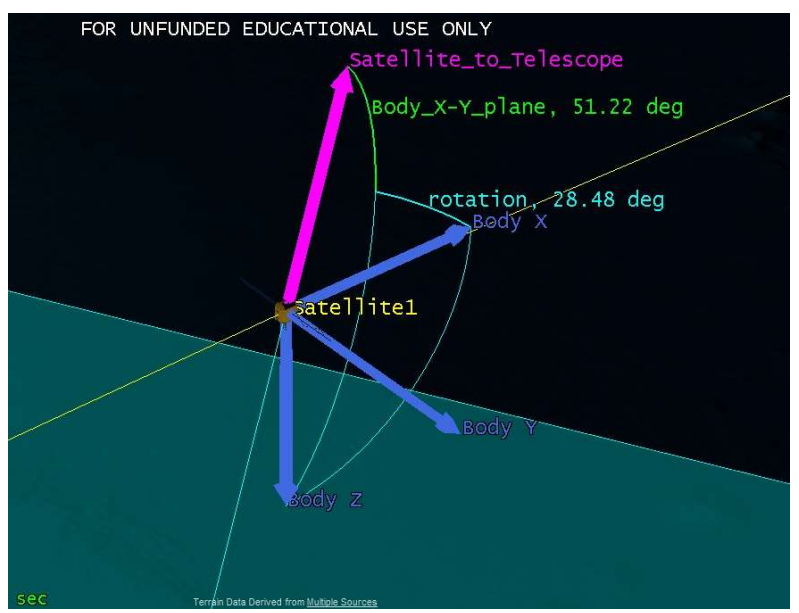

Figure 2: Target body coordinate system (blue arrows), telescope-to-target vector (pink arrow), rotation angles (cyan, green), orbital track (yellow line)

The telescope design selected for this simulation was a modified Cassegrain-type design with two mirrors and a refractive corrector near the image plane. Figure 3 shows the telescope model in FRED. The primary mirror is 74.7 $\mathrm{cm}$ in diameter with a central obscuration of $30.8 \mathrm{~cm}$ and $\mathrm{f} / 3$.7. The notional sensor array is an $\mathrm{HgCdTe}$ framing focal plane with $640 \times 480$ pixels and a pixel pitch of 25 $\mu \mathrm{m}$. The telescope/sensor combination delivers diffraction limited performance. An ISS-sized model was selected as an easy initial test case, as its large size ensures resolved imagery with the selected telescope design at closest approach.

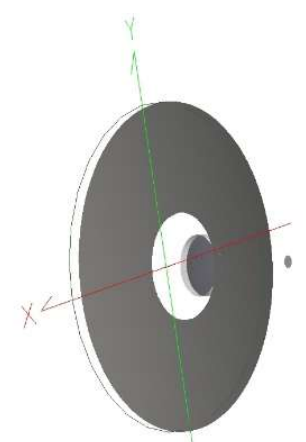

Figure 3: Telescope model in FRED 
The target was created as a simple 3-D model in FRED (Figure 4: Target model in FRED). Each surface was assigned a material: aluminum for target structural elements, indium gallium arsenide phosphide for the target solar panels, aluminum for the telescope mirror surfaces, and germanium for the telescope lens. Each surface was configured as an emitter in the LWIR band from 8 to $9 \mu \mathrm{m}$. Only rays which reach the simulated sensor array are traced.

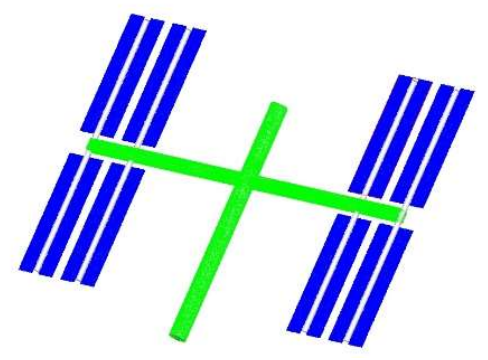

Figure 4: Target model in FRED

The target model was placed at a distance from the telescope corresponding to the target distance calculated by STK and rotated to correspond to the angle from which the telescope would view the target. For each target configuration FRED then generated an irradiance spread function across the sensor array, effectively a simulated thermal image (Figure 5,Figure 6), as well as polarization information for each pixel on the array. An embedded script takes that information and determines the aggregate Stokes vector for the target in its current configuration. As expected, the $s_{3}$ values were consistently 4 to 5 orders of magnitude weaker than $s_{0}$, $s_{1}$, and $s_{2}$.

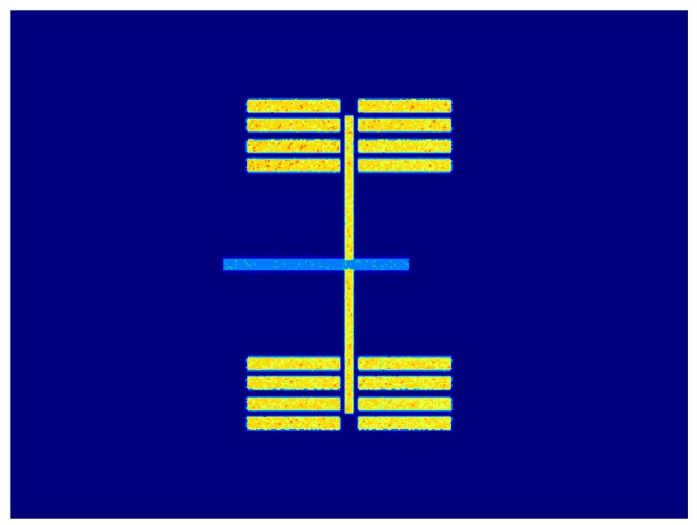

Figure 5: Simulated thermal image of target object

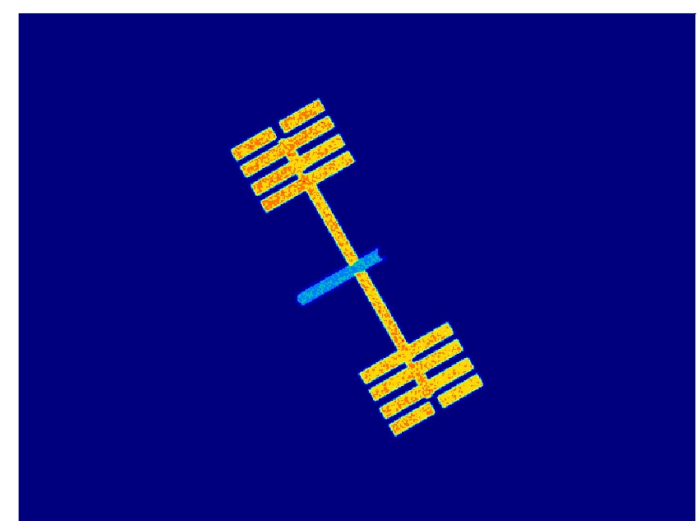

Figure 6: Simulated thermal image of target object rotated about its $y$ - and $z$-axes (60 degrees and 30 degrees, respectively)

Table 1 shows the results for the two target views presented in Figure 5 andFigure 6. Because the target features a rounded surface for part of its structure, even the straight-on view has some linear polarization. The angled target has a much higher value, however, as expected. Moving the target farther from the sensor reduces the magnitudes of the Stokes vector components, but not their relative values, so that DOLP is unchanged.

Table 1: Simulated polarization values for different rotation angles of the target $\left(s_{0}, s_{1}, s_{2}\right.$ in $\left.\mathrm{W} / \mathrm{m}^{2}\right)$

\begin{tabular}{|l|l|l|l|l|}
\hline & $s_{0}$ & $s_{1}$ & $s_{2}$ & DOLP \\
\hline Figure 5 & 0.0210 & $-2.54 \times 10^{-5}$ & 0.0145 & .694 \\
\hline Figure 6 & 0.0122 & 0.0103 & -0.00596 & .979 \\
\hline
\end{tabular}

\section{CONCLUSIONS}

Using a two advanced modeling tools, STK and FRED, it has been shown that it is possible to characterize a target at LEO distances and measure its polarization state using a simulated LWIR polarimeter.

The work so far is a proof of concept, showing that a LWIR polarimeter can be used to characterize remote space objects. Key questions for the continuing work on the subject are the range of target parameters for which this methodology works; i.e. how small a target, how far away, and to what degree individual objects can be characterized and identified, especially in a scene with multiple targets present. 


\section{REFERENCES}

1. Gurton, K., Felton, M., Mack, R., LeMaster, D., Farlow, C., Kudenov, M., Pezzaniti, L., "MidIR and LWIR Polarimetric Sensor Comparison Study," Proceedings of SPIE Defense, Security and Sensing: Polarization: Measurement, Analysis, and Remote Sensing IX, Orlando, FL, April 2010.

2. LeMaster, D., Eismann, M., "Passive Polarimetric Imaging," in Multi-Dimensional Imaging, John Wiley \& Sons, 2014.

3. Speicher, A., Matin, M., Tippets, R., Chun, F., "Calibration of a System to Collect Visible-Light Polarization Data for Classification of Geosynchronous Satellites," Proceedings of SPIE Optical Engineering + Applications: Remote Sensing System Engineering V, San Diego, CA, September 2014.

4. Speicher, A., Identification of Geostationary Satellites Using Polarization Data from Unresolved Images, University of Denver, Denver, 2015.

5. Beamer, D. K., Abeywickrema, U., Banerjee, P. P., "Statistical Analysis of Polarization Vectors for Target Identification," Optical Engineering 57(5), May 2018.

6. Beamer, D. K., Abeywickrema, U., Banerjee, P. P., "Statistical Analysis of Polarization Vectors for Target Identification," Proceedings of SPIE Optical Engineering + Applications: Polarization Science and Remote Sensing VIII, San Diego, CA, 2017

7. Rogne, T. J., Smith, F. G., Rice, J. E., "Passive Target Detection Using Polarized Components of Infrared Signatures," Proceedings of Polarimetry '90: Radar, Infrared, Visible, Ultraviolet, and XRay, Huntsville, AL, October 1990.

8. Wellems, D., Ortega, S.,Bowers, D., Boger, J., and Fetrow, M., "Long Wave Infrared Polarimetric Model: Theory, Measurements, and Parameters," Journal of Optics A: Pure and Applied Optics, vol. 8, September 2006.

9. Baddiley, C. J. "The Potential of CdHgTe Staring Array Infrared Detectors for Satellite Detection," Proceedings of Optical Systems for Space and Defence, London, United Kingdom, 1990.

10. Seniw, W. P., "LWIR Observations of Geosynchronous Satellites," Proceedings of the 1993 Space Surveillance Workshop, Lexington, MA, 1993.
11. Lee, J. K., and Nishimoto, D. L., "Infrared Detection of Geosynchronous Objects at AMOS," Proceedings of the 1993 Space Surveillance Workshop, Lexington, MA, 1993.

12. McCall, P. D., Naudeau, M. L., Adjouadi, M., "Debris Characterization Techniques Via Unresolved Long-Wave Infrared Imaging from a Space Platform," Journal of Applied Remote Sensing, 8(1), May 2014.

13. Fowles, G. R., Introduction to Modern Optics, Dover Publications, New York, 1989.

14. Azzam, R. M. A. and Bashara, N. M., Ellipsometry and Polarized Light, North-Holland Publishing Company, Amsterdam, 1977.

15. Chipman, R. A., "Polarimetry," in Handbook of Optics, Volume II, McGraw-Hill, New York, 1995. 